\title{
Detailed comparison of amyloid PET and CSF biomarkers for identifying early Alzheimer disease
}

OPEN

Sebastian Palmqvist, MD, $\mathrm{PhD}$

Henrik Zetterberg, MD, $\mathrm{PhD}$

Niklas Mattsson, MD, $\mathrm{PhD}$

Per Johansson, MD, PhD

For the Alzheimer's

Disease Neuroimaging Initiative

Lennart Minthon, MD, $\mathrm{PhD}$

Kaj Blennow, MD, PhD

Mattias Olsson, PhD

For the Swedish

BioFINDER study group

Oskar Hansson, MD, $\mathrm{PhD}$

Correspondence to Dr. Palmqvist: sebastian.palmqvist@med.lu.se or Dr. Hansson: oskar.hansson@med.lu.se

\section{Supplemental data} at Neurology.org

\section{ABSTRACT}

Objective: To compare the diagnostic accuracy of CSF biomarkers and amyloid PET for diagnosing early-stage Alzheimer disease (AD).

Methods: From the prospective, longitudinal BioFINDER study, we included 122 healthy elderly and 34 patients with mild cognitive impairment who developed AD dementia within 3 years (MCl-AD). $\beta$-Amyloid $(A \beta)$ deposition in 9 brain regions was examined with $\left[{ }^{18} \mathrm{~F}\right]$-flutemetamol PET. CSF was analyzed with INNOTEST and EUROIMMUN ELISAs. The results were replicated in 146 controls and 64 patients with MCl-AD from the Alzheimer's Disease Neuroimaging Initiative study.

Results: The best CSF measures for identifying MCI-AD were $A \beta 42 /$ total tau (t-tau) and $A \beta 42$ / hyperphosphorylated tau (p-tau) (area under the curve [AUC] 0.93-0.94). The best PET measures performed similarly (AUC 0.92-0.93; anterior cingulate, posterior cingulate/precuneus, and global neocortical uptake). CSF $A \beta 42 /$ t-tau and $A \beta 42 / p$-tau performed better than CSF A $\beta 42$ and $A \beta 42 / 40$ (AUC difference 0.03-0.12, $p<0.05$ ). Using nonoptimized cutoffs, CSF A $342 /$ t-tau had the highest accuracy of all CSF/PET biomarkers (sensitivity $97 \%$, specificity $83 \%$ ). The combination of CSF and PET was not better than using either biomarker separately.

Conclusions: Amyloid PET and CSF biomarkers can identify early AD with high accuracy. There were no differences between the best CSF and PET measures and no improvement when combining them. Regional PET measures were not better than assessing the global A $\beta$ deposition. The results were replicated in an independent cohort using another CSF assay and PET tracer. The choice between CSF and amyloid PET biomarkers for identifying early AD can be based on availability, costs, and doctor/patient preferences since both have equally high diagnostic accuracy.

Classification of evidence: This study provides Class III evidence that amyloid PET and CSF biomarkers identify early-stage AD equally accurately. Neurology ${ }^{\circledR}$ 2015;85:1240-1249

\section{GLOSSARY}

$\mathbf{A} \boldsymbol{\beta}=\beta$-amyloid $\mathbf{A D}=$ Alzheimer disease $\mathbf{A D N I}=$ Alzheimer's Disease Neuroimaging Initiative; $\mathbf{A U C}=$ area under the receiver operating characteristic curve; $\mathbf{C l}=$ confidence interval; $\mathbf{M C I}-\mathbf{A D}=$ mild cognitive impairment later developing into AD; $\mathbf{M C S}=$ mild cognitive symptoms; $\mathbf{M S D}=$ Meso Scale Discovery; $\mathbf{O R}=$ odds ratio; $\mathbf{p}$-tau = hyperphosphorylated tau; $\mathbf{R O C}=$ receiver operating characteristic; SUVR $=$ standardized uptake value ratio; $\mathbf{t}$-tau $=$ total tau; $\mathbf{V O I}=$ volume of interest; $\mathbf{Y I}=$ Youden index.

Biomarkers of cerebral $\beta$-amyloid $(A \beta)$ are used in the criteria for the early stages of Alzheimer disease $(\mathrm{AD}),{ }^{1,2}$ and are increasingly used in clinical trials. ${ }^{3-5}$ This stresses the need for reliable and available biomarkers of brain $A \beta$ pathology. Two $A \beta$ modalities have been established-CSF A $\beta 42$ and amyloid PET — which both correlate highly with brain biopsy findings. ${ }^{6,7}$ A potential advantage of amyloid PET over CSF A $\beta 42$ as an early diagnostic marker is the possibility to detect regional $\mathrm{A} \beta$ depositions that might occur before the global neocortical signal becomes pathologic. On the other hand, CSF analysis has the advantages that it may easily incorporate assessments

Authors' affiliations are provided at the end of the article.

Coinvestigators are listed on the Neurology ${ }^{\circledR}$ Web site at Neurology.org.

Part of the data used in preparation of this article were obtained from the Alzheimer's Disease Neuroimaging Initiative (ADNI) database (adni.loni. usc.edu). As such, the investigators within the ADNI contributed to the design and implementation of ADNI or provided data but did not participate in analysis or writing of this report.

Go to Neurology.org for full disclosures. Funding information and disclosures deemed relevant by the authors, if any, are provided at the end of the article. The Article Processing Charge was paid by the Swedish BioFINDER study.

This is an open access article distributed under the terms of the Creative Commons Attribution-NonCommercial-NoDerivatives License 4.0 (CC BY-NCND), which permits downloading and sharing the work provided it is properly cited. The work cannot be changed in any way or used commercially. 
such as tau (a measure of neuronal degenera$\operatorname{tion}^{8}$ ) and hyperphosphorylated tau (p-tau; a potential marker of tau pathology). ${ }^{9}$

Several studies have examined the agreement between amyloid PET and CSF $\mathrm{A} \beta 42,{ }^{10-22}$ but head-to-head studies comparing their diagnostic accuracy for incipient $\mathrm{AD}$ are scarce. Very few studies have used clinically relevant, consecutively recruited patients. To our knowledge, no previous study has compared the accuracy of regional amyloid PET and different CSF assays or ratios of CSF biomarkers such as $A \beta 42 / 40, A \beta 42 /$ total tau ( $\mathrm{t}$-tau), and $\mathrm{A} \beta 42 / \mathrm{p}$-tau when identifying cases with incipient $\mathrm{AD}$. We therefore performed a detailed head-to-head comparison of regional and global amyloid PET and CSF analysis with 2 different assays in a clinical cohort of consecutive patients with mild cognitive impairment who later developed $\mathrm{AD}$ dementia (MCI-AD). We also examined the diagnostic benefit of combining CSF and PET measures.

METHODS This study conducts a head-to-head comparison of the diagnostic accuracy of amyloid PET and CSF biomarkers for identifying early-stage $\mathrm{AD}$. It provides Class III evidence that amyloid PET and CSF biomarkers identify early-stage AD equally accurately.

Subjects. The present study population was part of the prospective and longitudinal Swedish BioFINDER study, which, among other cohorts, consecutively enrolls patients without dementia with mild cognitive symptoms (MCS) from 3 participating memory clinics in Sweden. More information about the design and populations is available at biofinder.se and in the online supplement (e-Methods on the Neurology ${ }^{\circledR}$ Web site at Neurology.org). We included patients with MCS who had progressed to AD dementia during the follow-up period (hereafter referred to as MCI-AD). This resulted in a sample of 34 patients with MCI-AD. The mean follow-up time was 2.0 years (range 0.8-3.4). A consensus group (Katarina Nägga, P.J., S.P.) determined the follow-up diagnosis probable $\mathrm{AD}^{23}$ in September 2014. The group was blinded to all biomarker data. At baseline in the MCS cohort, 3 patients (9\%) had subjective cognitive decline and 31 (91\%) had MCI (48\% amnestic single-domain, 39\% amnestic multidomain, and 12\% nonamnestic). A total of 122 cognitively healthy elderly from the BioFINDER study were included as controls. ${ }^{24}$

Standard protocol approvals, registrations, and patient consents. The Ethics Committee in Lund, Sweden, approved the study. All patients gave their written informed consent.

Amyloid PET scanning and analysis. Cerebral $\mathrm{A} \beta$ deposition was visualized with the PET tracer ${ }^{18} \mathrm{~F}$-flutemetamol (approved by the Food and Drug Administration and the European Medical Agency). ${ }^{25}$ PET/CT scanning of the brain was conducted at 2 sites using the same type of scanner (Gemini, Philips Healthcare, Best, the Netherlands). Baseline sum images from 90-110 minutes postinjection were analyzed using the software NeuroMarQ (GE Healthcare, Cleveland, OH). A volume of interest (VOI) template was applied for the following 9 bilateral regions: prefrontal, parietal, lateral temporal, medial temporal, sensorimotor, occipital, anterior cingulate, posterior cingulate/precuneus, and a global neocortical composite region. ${ }^{26}$ The standardized uptake value ratio (SUVR) was defined as the uptake in a VOI normalized for the cerebellar cortex uptake.

CSF analysis. The procedure and analysis of the CSF followed the Alzheimer's Association Flow Chart for CSF biomarkers. ${ }^{8}$ Baseline lumbar CSF samples were collected at the 3 centers and analyzed at one center on one occasion using single batch analysis according to a standardized protocol. ${ }^{8,20}$ CSF $\mathrm{t}$-tau, $A \beta 40$, and $A \beta 42$ were analyzed by EUROIMMUN (EI) ELISAs (EUROIMMUN AG, Lübeck, Germany). CSF A $\beta 42$ and tau phosphorylated at Thr181 (p-tau) were analyzed with INNOTEST ( ${ }^{(T)}$ ELISAs (Fujirebio Europe, Ghent, Belgium). The following 8 variables were derived from the CSF analyses: $\mathrm{A} \beta 42^{\mathrm{IT}}, A \beta 42^{\mathrm{EI}}, A \beta 42^{\mathrm{IT}} / \mathrm{A} \beta 40^{\mathrm{EI}}, A \beta 42^{\mathrm{IT}} / \mathrm{t}-\mathrm{tau}^{\mathrm{EI}}, A \beta 42^{\mathrm{IT}} /$ p-tau ${ }^{\mathrm{IT}}, \mathrm{A} \beta 42^{\mathrm{EI}} / \mathrm{A} \beta 40^{\mathrm{EI}}, \mathrm{A} \beta 42^{\mathrm{EI}} / \mathrm{p}-\mathrm{tau}^{\mathrm{IT}}, \mathrm{A} \beta 42^{\mathrm{EI}} / \mathrm{t}-\mathrm{tau}^{\mathrm{EI}}$.

Hippocampus volume and cognition. All patients were examined using a single 3T MRI scanner (Trio, Siemens, Munich, Germany). Hippocampal volume was analyzed with FreeSurfer version 5.1. The smallest hippocampal volume (left or right) was used. Global cognition was assessed with the Mini-Mental State Examination. Memory was assessed with the 10-word delayed recall test from the Alzheimer's Disease Assessment Scale-cognitive subscale. ${ }^{27}$

Alzheimer's Disease Neuroimaging Initiative cohort. To validate the results from BioFINDER in an independent cohort, we used data from the Alzheimer's Disease Neuroimaging Initiative (ADNI; adni.loni.usc.edu). The sample consisted of 64 patients with MCI-AD and 146 controls who had undergone CSF sampling and A $\beta$ PET at baseline of ADNI-2 (table 1 and reference 19). In sum, the PET tracer ${ }^{18} \mathrm{~F}$-florbetapir was used to quantify $A \beta$ in different brain regions (and globally), normalized for the cerebellar uptake. CSF $A \beta 42, t$-tau, and $p$-tau were measured using xMAP Luminex (Luminex Corp., Austin, TX) with the INNOBIA AlzBio3 kit (Innogenetics, Ghent, Belgium) ${ }^{28} \mathrm{~A}$ consensus group blinded to the biomarker data determined the follow-up diagnoses.

Statistical analysis. Group differences were calculated with the Mann-Whitney $U$ test (table 1 ). The area under the receiver operating characteristic (ROC) curve (AUC) was used to examine the diagnostic accuracy of the continuous CSF and PET variables (table 2). The $95 \%$ confidence interval (CI) and significance for differences between the AUCs were calculated using bootstrap techniques. ${ }^{29}$ The AUCs of the combined CSF and PET variables were derived from logistic regressions. Nonoptimized and unbiased cutoffs were established using mixture modeling. ${ }^{30}$ A Youden index (YI; sensitivity + specificity - 1) was used for an easier comparison of sensitivities and specificities. Odds ratios (OR) were calculated with multivariate logistic regression analysis (table 3). The statistical analyses were performed with MedCalc version 14 (MedCalc Software, MariaKerke, Belgium); SPSS, version 22.0 (SPSS Inc., Chicago, IL); MATLAB release 2014, Statistics Toolbox (MathWorks, Natick, MA); and $R$ version 3.0.2.

RESULTS Baseline characteristics are shown in table 1. There were no significant differences in age, APOE4, or sex between the ADNI and BioFINDER cohorts, but education differed between control and MCI-AD populations (higher in ADNI, $p<0.001$, 
Table 1 Baseline characteristics of MCI-AD patients and cognitively healthy elderly from the BioFINDER study and the ADNI study

\begin{tabular}{|c|c|c|c|c|c|c|}
\hline & $\begin{array}{l}\text { BioFINDER MCI-AD } \\
(n=34)\end{array}$ & $\begin{array}{l}\text { BioFINDER controls } \\
(n=122)\end{array}$ & $\begin{array}{l}\text { p Value BioFINDER } \\
\text { MCl-AD vs controls }\end{array}$ & $\begin{array}{l}\text { ADNI MCI-AD } \\
(n=64)\end{array}$ & $\begin{array}{l}\text { ADNI controls } \\
(n=146)\end{array}$ & $\begin{array}{l}p \text { Value ADNI } \\
\text { MCI-AD vs controls }\end{array}$ \\
\hline \multicolumn{7}{|l|}{ Demographics } \\
\hline Age, y (range) & $72.7(63-80)$ & 73.5 (65-85) & 0.29 & $72.1(48-85)$ & $73.2(56-89)$ & 0.79 \\
\hline Female, \% & 54 & 64 & 0.60 & 45 & 52 & 0.42 \\
\hline Education, y & $11.9 \pm 3.8$ & $11.3 \pm 3.3$ & 0.67 & $16.0 \pm 2.8$ & $16.6 \pm 2.5$ & 0.13 \\
\hline APOE $\varepsilon 4, \geq 1$ allele, $\%$ & 61 & 24 & $<0.001$ & 73 & 27 & $<0.001$ \\
\hline MMSE, points & $26.7 \pm 1.5$ & $29.0 \pm 0.9$ & $<0.001$ & $27.0 \pm 1.8$ & $29.1 \pm 1.2$ & $<0.001$ \\
\hline $\begin{array}{l}\text { 10-word delayed recall, } \\
\text { errors }\end{array}$ & $7.2 \pm 2.3$ & $2.0 \pm 2.0$ & $<0.001$ & & & \\
\hline Hippocampus volume, $\mathrm{cm}^{3}$ & $3.1 \pm 0.5$ & $3.6 \pm 0.4$ & $<0.001$ & & & \\
\hline \multicolumn{7}{|l|}{ PET regions } \\
\hline Global/composite & $2.11 \pm 0.47$ & $1.29 \pm 0.28$ & $<0.001$ & & & \\
\hline Prefrontal & $2.11 \pm 0.49$ & $1.24 \pm 0.31$ & $<0.001$ & & & \\
\hline Anterior cingulate & $2.36 \pm 0.52$ & $1.41 \pm 0.34$ & $<0.001$ & & & \\
\hline $\begin{array}{l}\text { Posterior cingulate/ } \\
\text { precuneus }\end{array}$ & $2.26 \pm 0.48$ & $1.39 \pm 0.33$ & $<0.001$ & & & \\
\hline Parietal & $1.98 \pm 0.44$ & $1.23 \pm 0.26$ & $<0.001$ & & & \\
\hline Lateral temporal & $2.14 \pm 0.50$ & $1.39 \pm 0.25$ & $<0.001$ & & & \\
\hline Medial temporal & $1.58 \pm 0.29$ & $1.36 \pm 0.16$ & $<0.001$ & & & \\
\hline Occipital & $1.83 \pm 0.40$ & $1.37 \pm 0.19$ & $<0.001$ & & & \\
\hline Sensorimotor & $1.81 \pm 0.42$ & $1.31 \pm 0.18$ & $<0.001$ & & & \\
\hline \multicolumn{7}{|l|}{ CSF analyses } \\
\hline$A \beta 42^{E I}$ & $333 \pm 114$ & $538 \pm 186$ & $<0.001$ & & & \\
\hline$A \beta 42^{\prime T}$ & $380 \pm 102$ & $659 \pm 184$ & $<0.001$ & & & \\
\hline$A \beta 40^{E I}$ & $4,881 \pm 1,877$ & $4,516 \pm 1,522$ & 0.47 & & & \\
\hline$t-\operatorname{tau}^{E I}$ & $581 \pm 213$ & $318 \pm 113$ & $<0.001$ & & & \\
\hline p-tau ${ }^{\prime T}$ & $92.4 \pm 33.9$ & $53.5 \pm 18.0$ & $<0.001$ & & & \\
\hline A $\beta 42^{E I} / A \beta 40^{E I}$ & $0.072 \pm 0.024$ & $0.12 \pm 0.038$ & $<0.001$ & & & \\
\hline$A \beta 42^{\prime T} / A \beta 40^{E I}$ & $0.086 \pm 0.034$ & $0.16 \pm 0.052$ & $<0.001$ & & & \\
\hline$A \beta 42^{E I} / t-t^{E}{ }^{E I}$ & $0.63 \pm 0.27$ & $1.87 \pm 0.76$ & $<0.001$ & & & \\
\hline$A \beta 42^{E 1} / p-\operatorname{tau}^{\prime T}$ & $3.96 \pm 1.73$ & $11.0 \pm 4.42$ & $<0.001$ & & & \\
\hline$A \beta 42^{1 T} / t-\operatorname{tau}^{E I}$ & $0.73 \pm 0.33$ & $2.31 \pm 0.89$ & $<0.001$ & & & \\
\hline$A \beta 42^{\prime T} / p-\operatorname{tau}^{\prime T}$ & $4.59 \pm 2.0$ & $13.7 \pm 5.39$ & $<0.001$ & & & \\
\hline
\end{tabular}

Abbreviations: $A D N I=$ Alzheimer's Disease Neuroimaging Initiative; EI = EUROIMMUN assay; IT = INNOTEST assay; MCI-AD = patients with mild cognitive impairment who developed Alzheimer disease within 3 years; MMSE = Mini-Mental State Examination; $p$-tau = hyperphosphorylated tau; $t$-tau = total tau. Biomarker data of the replication population (ADNI study) can be found in table e-1. As for comparisons between demographics in the BioFINDER and ADNI cohorts, only education differed significantly $(p<0.001)$. Values are mean $\pm \mathrm{SD}$, unless otherwise specified. CSF measures are given in $\mathrm{pg} / \mathrm{mL}$ and PET score in mean standardized uptake value ratio.

table 1). Biomarker data could not be directly compared between the studies because of different CSF assays and PET tracers (table 1 and table e-1).

CSF biomarkers for classification of MCI-AD and controls. The CSF biomarkers had diagnostic accuracies for MCI-AD ranging from AUC 0.82 (CSF $\mathrm{A} \beta 42^{\mathrm{EI}}$ ) to AUC 0.93-0.94 (CSF A $342 / \mathrm{t}$-tau and $\mathrm{A} \beta 42 / \mathrm{p}$-tau ratios independent of assay; table 2). CSF $A \beta 42^{\mathrm{IT}} / \mathrm{t}-\operatorname{tau}^{\mathrm{EI}}$ and $\mathrm{A} \beta 42^{\mathrm{IT}} / \mathrm{p}-\mathrm{tau}^{\mathrm{IT}} \mathrm{had}$ significantly better accuracies than CSF $A \beta 42^{\mathrm{IT}}$
(AUC difference: $0.04-0.05, p=0.02$ ) and $\mathrm{A} \beta 42^{\mathrm{EI}} / \mathrm{A} \beta 40^{\mathrm{EI}}$ (AUC difference: 0.08, $p<0.001$ ). CSF $A \beta 42^{\mathrm{EI}}$ had significantly lower AUC compared to most other biomarkers, but this could be partly overcome by the ratio of $\mathrm{A} \beta 42^{\mathrm{EI}} / \mathrm{A} \beta 40^{\mathrm{EI}}$. The diagnostic accuracy of CSF $A \beta 42^{\mathrm{IT}}$, on the other hand, was not improved when used as a ratio with $\mathrm{A} \beta 40^{\mathrm{EI}}$ (table 2).

Regional and composite PET biomarkers for classification of MCI-AD vs controls. The AUCs of the amyloid PET biomarkers ranged from 0.75 to 
Table 2 Classification of MCI-AD and healthy controls in BioFINDER based on ROC analyses in BioFINDER

\begin{tabular}{|c|c|c|}
\hline $\begin{array}{l}\text { Variable (in order of AUC } \\
\text { value) }\end{array}$ & AUC (95\% CI) & AUC significantly better than \\
\hline CSF A $\beta 42^{\prime T} / p-$ tau $^{\prime T}$ & $0.94(0.89-0.97)$ & $\begin{array}{l}\text { PET medial temporal, PET occipital, CSF } \\
A \beta 42^{E I} / A \beta 40^{E I}, A \beta 42^{E I}, A \beta 42^{I T}\end{array}$ \\
\hline $\operatorname{CSF} A \beta 42^{E I} / t-\operatorname{tau}^{E I}$ & $0.93(0.88-0.97)$ & $\begin{array}{l}\text { PET medial temporal, CSF A } \beta 42^{\mathrm{E} I} / \mathrm{A} \beta 40^{\mathrm{EI}} \text {, } \\
\mathrm{A} \beta 42^{\mathrm{EI}}\end{array}$ \\
\hline CSF A $\beta 42^{I T} /$ t-tau ${ }^{E I}$ & $0.93(0.88-0.97)$ & $\begin{array}{l}\text { PET medial temporal, PET occipital, CSF } \\
A \beta 42^{E I} / A \beta 40^{E I}, A \beta 42^{E I}, A \beta 42^{I T}\end{array}$ \\
\hline CSF A $\beta 42^{E I} / p-$ tau $^{\prime T}$ & $0.93(0.88-0.96)$ & $\begin{array}{l}\text { PET medial temporal, CSF } A \beta 42^{\mathrm{E} I} / \mathrm{A} \beta 40^{\mathrm{EI}} \text {, } \\
\mathrm{A} \beta 42^{\mathrm{EI}}\end{array}$ \\
\hline $\begin{array}{l}\text { PET posterior cingulate/ } \\
\text { precuneus }\end{array}$ & $0.93(0.87-0.96)$ & $\begin{array}{l}\text { PET medial temporal, PET occipital, PET } \\
\text { sensorimotor, CSF A } 342^{\mathrm{EI}}\end{array}$ \\
\hline PET anterior cingulate & $0.92(0.87-0.96)$ & $\begin{array}{l}\text { PET medial temporal, PET occipital, CSF } \\
\text { A } \beta 42^{E I}\end{array}$ \\
\hline PET composite & $0.92(0.86-0.95)$ & PET medial temporal, PET occipital \\
\hline PET prefrontal & $0.91(0.86-0.95)$ & PET medial temporal, CSF $A \beta 42^{\mathrm{EI}}$ \\
\hline PET parietal & $0.91(0.85-0.95)$ & $\begin{array}{l}\text { PET medial temporal, PET occipital, PET } \\
\text { sensorimotor }\end{array}$ \\
\hline $\operatorname{CSF} A \beta 42^{\prime T}$ & $0.90(0.84-0.94)$ & PET medial temporal, CSF $A \beta 42^{E I}$ \\
\hline PET lateral temporal & $0.90(0.84-0.94)$ & PET medial temporal, PET occipital \\
\hline CSF $A \beta 42^{\prime T} / A \beta 40^{E I}$ & $0.88(0.80-0.94)$ & PET medial temporal \\
\hline CSF $A \beta 42^{\mathrm{EI}} / \mathrm{A} \beta 40^{\mathrm{EI}}$ & $0.86(0.80-0.91)$ & PET medial temporal \\
\hline PET sensorimotor & $0.85(0.79-0.90)$ & PET medial temporal \\
\hline PET occipital & $0.84(0.77-0.89)$ & PET medial temporal \\
\hline CSF A $\beta 42^{\mathrm{EI}}$ & $0.82(0.74-0.89)$ & \\
\hline PET medial temporal & 0.75 (0.68-0.82) & \\
\hline
\end{tabular}

Abbreviations: $\mathrm{AUC}=$ area under the curve; $\mathrm{Cl}=$ confidence interval; $\mathrm{EI}=\mathrm{EUROIMMUN}$ assay; IT = INNOTEST assay; $\mathrm{MCl}-\mathrm{AD}=$ patients with mild cognitive impairment who developed Alzheimer disease within 3 years; $\mathrm{p}$-tau = hyperphosphorylated tau; ROC = receiving operating characteristic; $\mathrm{t}$-tau $=$ total tau.

AUC was calculated with ROC analysis. The $95 \% \mathrm{Cl}$ and significance for differences between the AUCs were calculated using bootstrap techniques with 5,000 bootstrap replicas.
Combination of CSF and PET biomarkers. To examine the potential benefit of combining PET and CSF analysis, we tested models with CSF $A \beta 42^{\mathrm{IT}} / \mathrm{p}-\mathrm{tau}^{\mathrm{IT}}$ and the composite PET SUVR entered separately and together as predictors of diagnosis in logistic regression analyses. When used together, the AUC was 0.96 (95\% CI 0.92-0.97) and both variables were independent significant predictors $(p<0.01)$. This was numerically higher than for models using the individual modalities, but the differences were not significant (AUC difference 0.021-0.047, $p=$ 0.07-0.08). A combined model of the composite PET SUVR and CSF $\mathrm{p}-\mathrm{tau}^{\mathrm{TT}}$ had equal AUC value as CSF $\mathrm{A} \beta 42^{\mathrm{IT}} / \mathrm{p}^{- \text {tau }^{\mathrm{IT}}}$ (both were 0.94, 95\% CI 0.89-0.97).

Classification of incipient $\mathrm{AD}$ and controls at specific cutoffs. All $A \beta$ variables had a bimodal distribution suitable for establishing nonoptimized, unbiased cutoffs with mixture modeling except for CSF $\mathrm{A} \beta 42^{\mathrm{EI}}$, which was excluded from this analysis. When using these cutoffs in the ROC analysis, CSF A $\beta 42^{\mathrm{IT}} / \mathrm{t}-\mathrm{tau}^{\mathrm{EI}}$ and CSF $A \beta 42^{\mathrm{IT}} / \mathrm{p}$-tau ${ }^{\mathrm{IT}}$ had the best sensitivities and specificities of all CSF and PET measures (table 3). The 2 best PET measures were the prefrontal and the posterior cingulate/precuneus regions. Scatterplots show that the differences in specificities between CSF and PET are mostly caused by controls with normal PET and abnormal CSF values (figures e- 1 and e-2). In logistic regressions, CSF $A \beta 42 / t-t^{I T}$ and EI had the highest OR, when adjusting for age, sex, memory function, $A P O E \varepsilon 4$, and hippocampal volume (table 3).

The diagnostic accuracy of biomarkers used in the clinic should preferably not be very sensitive to smaller changes in cutoff values, if they are to be generalizable between different centers and settings. In figure $1, \mathrm{~A}$ and $\mathrm{B}$, continuous sensitivities and specificities of 4 CSF and PET measures are shown as a function of the cutoff point. The CSF and PET measures were not dependent on an optimized cutoff but provide high accuracies from cutoff values spanning at least $1 \mathrm{SD}$ in the current sample. The exception was CSF $\mathrm{A} \beta 42^{\mathrm{EI}} / 40^{\mathrm{EI}}$, which had a slightly narrower interval with near optimal YI.

Comparison with the ADNI data. Accuracies for CSF and PET biomarkers were also analyzed in the independent ADNI cohort (table 4). All CSF and PET variables had similar AUCs ranging from 0.86 to 0.87 and no significant differences were found $(p=0.17-$ 0.93). As in BioFINDER, CSF $A \beta 42 / t$-tau and $\mathrm{A} \beta 42 / \mathrm{p}$-tau had higher AUCs than CSF A $\beta 42$ alone (both 0.87 vs 0.85 ), but in ADNI the differences were not significant $(p=0.60-0.65)$. In ADNI, the AUCs of t-tau $(0.81,95 \%$ CI $0.74-0.88)$ and p-tau $(0.82,95 \%$ CI $0.75-0.88)$ were lower than in 
Table $3 \quad$ ROC analysis in BioFINDER of MCI-AD and healthy controls based on unbiased cutoffs

\begin{tabular}{|c|c|c|c|c|c|}
\hline Variable (in order of Youden index) & Unbiased cutoff ${ }^{a}$ & Youden index $(95 \% \mathrm{Cl})^{\mathrm{b}, \mathrm{c}}$ & Sensitivity (\%) ${ }^{b}$ & Specificity (\%) $)^{b}$ & Adjusted OR $(95 \% \mathrm{Cl})^{\mathrm{d}}$ \\
\hline 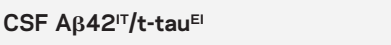 & 1.25 & $0.80(0.68-0.87)$ & 97 & 83 & $62(4.9-796)$ \\
\hline CSF A $\beta 42^{\prime T} / p-$ tau $^{\prime T}$ & 7.24 & $0.79(0.66-0.87)$ & 94 & 85 & $34(4.4-263)$ \\
\hline PET prefrontal & 1.48 & $0.74(0.56-0.84)$ & 88 & 86 & 29 (3.6-239) \\
\hline CSF A $\beta 42^{E I} / t-\operatorname{tau}^{E I}$ & 0.96 & $0.73(0.59-0.83)$ & 91 & 82 & $44(4.3-447)$ \\
\hline PET anterior cingulate & 1.62 & $0.72(0.56-0.81)$ & 91 & 80 & $22(2.9-170)$ \\
\hline PET composite & 1.51 & $0.72(0.55-0.83)$ & 85 & 87 & 29 (3.6-239) \\
\hline PET lateral temporal & 1.58 & $0.71(0.54-0.83)$ & 85 & 86 & $30(3.6-250)$ \\
\hline PET parietal & 1.43 & $0.70(0.53-0.81)$ & 85 & 84 & 25 (3.2-190) \\
\hline CSF Aß42IT/A $\beta 40^{E I}$ & 0.10 & $0.59(0.39-0.73)$ & 76 & 83 & $8.2(1.7-40)$ \\
\hline PET occipital & 1.68 & $0.49(0.30-0.66)$ & 56 & 93 & $27(2.8-263)$ \\
\hline PET medial temporal & 1.69 & $0.20(0.07-0.38)$ & 23 & 97 & Nonsignificant \\
\hline
\end{tabular}

Abbreviations: $\mathrm{Cl}$ = confidence interval; EI = EUROIMMUN assay; IT = INNOTEST assay; $\mathrm{MCI}-\mathrm{AD}$ = patients with mild cognitive impairment who developed Alzheimer disease within 3 years; OR = odds ratio; $\mathrm{p}$-tau $=$ hyperphosphorylated tau; $\mathrm{ROC}=$ receiving operating characteristic; $\mathrm{t}$-tau $=$ total tau .

Area under the curve was calculated with ROC analysis. The $95 \% \mathrm{Cl}$ and significance for differences between the AUCs were calculated using bootstrap techniques with 5,000 bootstrap replicas. PET values are shown in standardized uptake value ratio and CSF levels in pg/mL (except for the CSF ratios). ${ }^{a}$ Established with mixture modeling analysis (described in Methods).

${ }^{\mathrm{b}}$ Based on the ROC analysis (not derived from the logistic regression analysis).

${ }^{c}$ Youden index (sensitivity + specificity -1 ) is provided for an easier comparison of the combined value of the sensitivity and specificity, e.g., the diagnostic accuracy of CSF and PET measures as dichotomized variables. The value is based on the unbiased cutoffs.

${ }^{\mathrm{d}} \mathrm{A}$ logistic regression analysis was performed with the diagnosis (MCI-AD or control) as the dependent variable and the dichotomized CSF/PET variable as a covariate to yield an OR. Age, sex, APOE $\varepsilon 4$ allele, memory function, and hippocampal volume were adjusted for in the model.

BioFINDER (t-tau $0.88,95 \%$ CI $0.82-0.93$ and p-tau 0.87 , 95\% CI $0.80-0.91$; data not shown in the tables). However, no significant differences could be tested since the results were derived from 2 different cohorts.

When combining CSF A $\beta 42 / p$-tau and composite PET SUVR in ADNI, the AUC was 0.87 (95\% CI 0.82-0.93). This did not differ significantly from using the variables separately (AUC difference: $0.00-0.01 ; p=0.40-0.53)$.

DISCUSSION The main finding of this study was that the diagnostic accuracy of CSF and A $\beta$ PET biomarkers to identify MCI-AD was similar when using ${ }^{18}$ F-flutemetamol amyloid PET and several different CSF biomarkers. Specifically, the best CSF measures (CSF A $\beta 42 / \mathrm{t}$-tau and $\mathrm{A} \beta 42 / \mathrm{p}$-tau ratios) had similar diagnostic accuracies as the best PET measures (composite and cingulate SUVRs; table 2). We also found that no regional PET biomarker was better than the neocortical composite PET SUVR (table 2). For CSF biomarkers, the CSF A $342 / t$-tau or $A \beta 42 / p$-tau ratios had significantly higher diagnostic accuracy compared to using CSF $A \beta$ biomarkers alone. When using unbiased cutoffs,
CSF A $42 /$ t-tau had the highest sensitivity and specificity of all CSF and PET biomarkers (table 3). Finally, the combination of the best CSF and PET biomarkers did not provide any added diagnostic value compared to using either modality separately. The overall results were replicated in an independent cohort (ADNI).

Although we found that CSF $A \beta 42^{\mathrm{IT}}$ and $\mathrm{A} \beta 42^{\mathrm{EI}} /$ $\mathrm{A} \beta 40^{\mathrm{EI}}$ performed similarly to the best SUVRs of ${ }^{18}$ F-flutemetamol PET in terms of AUCs (table 2), these CSF biomarkers generally had lower specificities than the PET biomarkers when using unbiased cutoffs (table 3). The addition of $\mathrm{t}$-tau or $\mathrm{p}$-tau to $\mathrm{A} \beta 42$ (as ratios) significantly increased the diagnostic accuracy of CSF biomarkers (table 2). This supports the common usage of CSF $A \beta 42$ in combination with $t-$ tau or p-tau in clinical practice, and is in agreement with previous studies. ${ }^{31-34}$

The diagnostic accuracy of CSF A 342 was lower for EUROIMMUN compared with INNOTEST (table 2 and figure e-3). This was partly overcome by using the $A \beta 42 / 40$ ratio, which did not improve the accuracy of A 342 INNOTEST (table 2). This finding has not been shown previously and needs to be replicated in future studies, since the causes are 

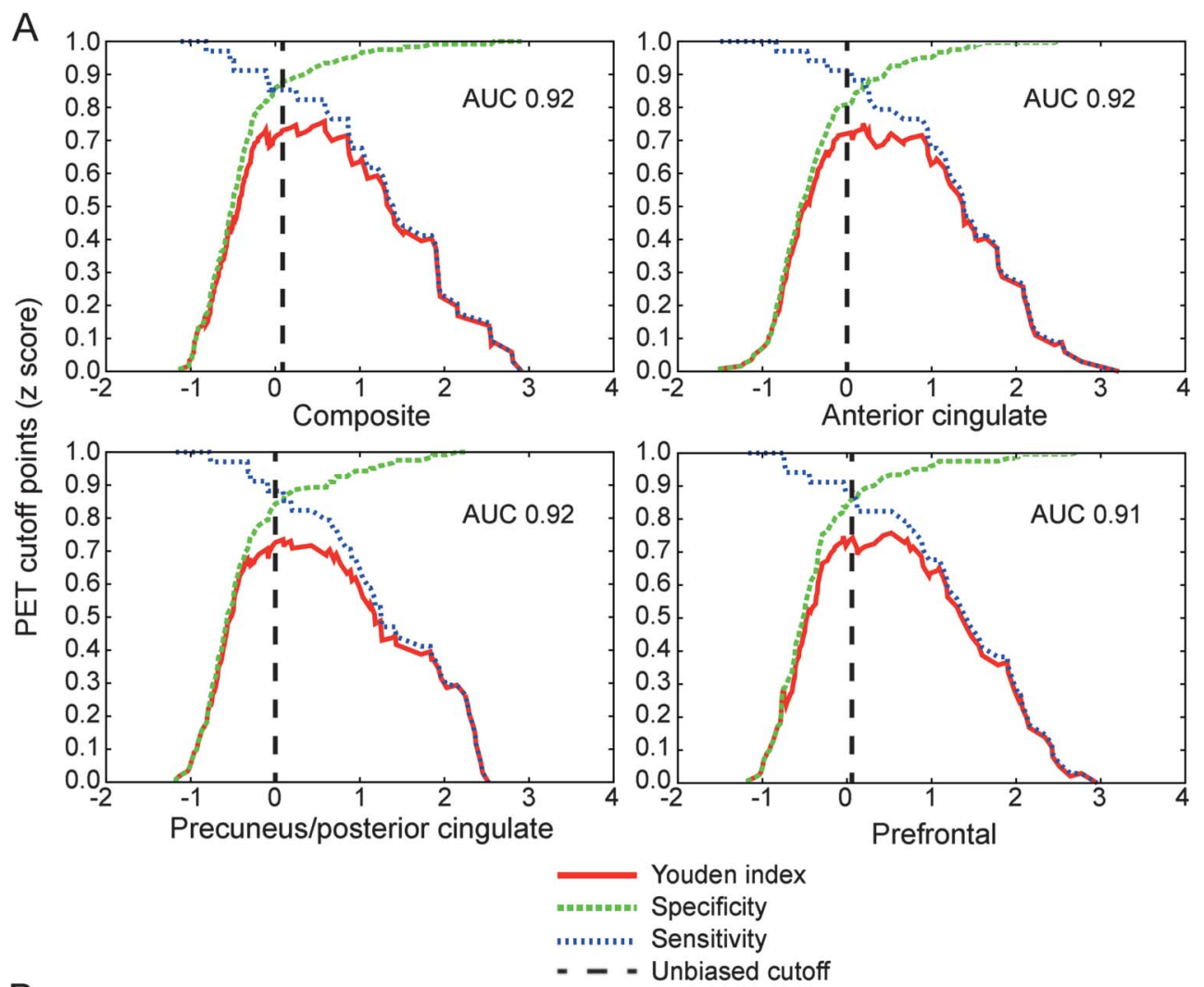

B

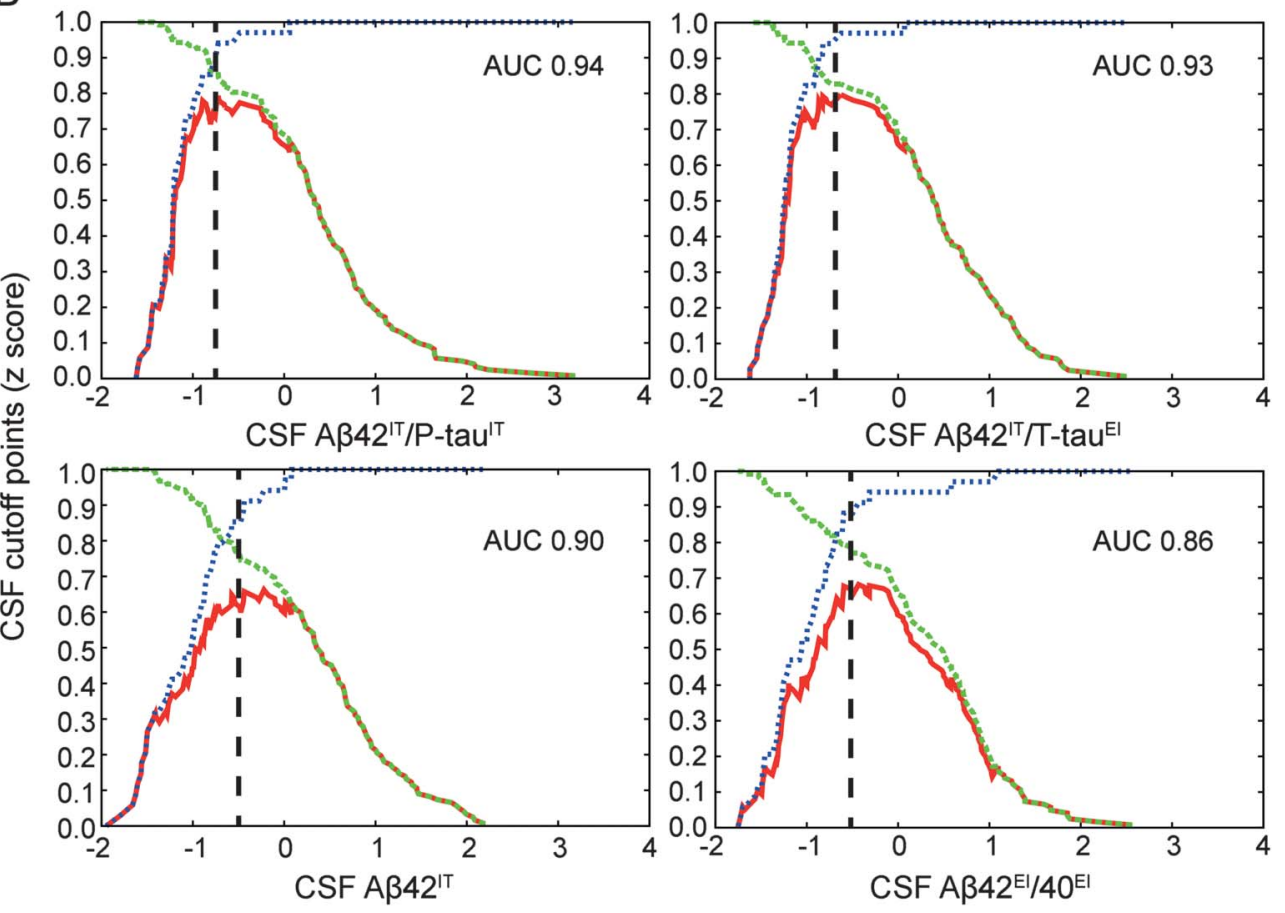

(A, B) The cutoffs have been transformed to $z$ scores (SD) for easier comparison between variables. Near optimal Youden indices are found for a relatively wide range of cutoffs ( $>1$ SD) for all variables except for CSF $A \beta 42 / A \beta 40^{E I}$, which have a slightly narrower span. The different cutoffs within this near optimal range thus only change the relationship between the sensitivity and specificity, but not the overall classification accuracy. This wide range of near optimal cutoffs suggests that the cutoffs are likely to produce high diagnostic accuracies in other populations. AUC = area under the receiver operating characteristic curve.

unknown. Few studies have compared different ELISAs for CSF A $\beta 42$ to identify MCI-AD. Hertze et al. ${ }^{33}$ found that CSF $A \beta 42$ analyzed with xMAP
AlzBio3 had higher diagnostic accuracy compared with the Meso Scale Discovery (MSD) assay, but this was overcome by using the $A \beta 42 / A \beta 40$ MSD ratio. 
Table 4 Classification of MCI-AD and healthy controls based on ROC analyses in ADNI

\begin{tabular}{|c|c|c|c|c|c|}
\hline Variable (in order of AUC value) & AUC $(95 \% \mathrm{Cl})$ & Unbiased cutoffa $^{a}$ & Youden index $(95 \% \mathrm{Cl})^{\mathrm{b}, \mathrm{c}}$ & Sensitivity $(\%)^{b}$ & Specificity $(\%)^{b}$ \\
\hline PET frontal & $0.87(0.82-0.91)$ & 1.13 & $0.63(0.51-0.73)$ & 89 & 74 \\
\hline CSF A $342 / t-t a u$ & $0.87(0.80-0.92)$ & 1.71 & $0.65(0.53-0.76)$ & 80 & 86 \\
\hline CSF Aß42/p-tau & $0.87(0.80-0.92)$ & 3.83 & $0.65(0.52-0.75)$ & 84 & 81 \\
\hline PET composite & $0.86(0.81-0.91)$ & 1.13 & $0.66(0.54-0.75)$ & 91 & 75 \\
\hline PET parietal & $0.86(0.81-0.91)$ & 1.11 & $0.59(0.47-0.69)$ & 91 & 68 \\
\hline PET temporal & $0.86(0.80-0.92)$ & 1.08 & $0.66(0.54-0.76)$ & 89 & 77 \\
\hline PET cingulate & $0.86(0.80-0.91)$ & 1.20 & $0.56(0.44-0.66)$ & 89 & 66 \\
\hline CSF A 342 & $0.85(0.79-0.90)$ & 173 & $0.60(0.50-0.70)$ & 94 & 66 \\
\hline
\end{tabular}

Abbreviations: $\mathrm{ADNI}=$ Alzheimer's Disease Neuroimaging Initiative; $\mathrm{AUC}=$ area under the receiver operating characteristic curve; $\mathrm{Cl}=$ confidence interval; $\mathrm{MCl}-\mathrm{AD}=$ patients with mild cognitive impairment who developed Alzheimer disease within 3 years; $\mathrm{p}$-tau $=$ hyperphosphorylated tau; $\mathrm{ROC}=$ receiving operating characteristic; $\mathrm{t}$-tau $=$ total tau.

No significant differences between the AUCs were found ( $p=0.17-0.93$ ).

${ }^{a}$ Established with mixture modeling analysis (described in Methods).

${ }^{\mathrm{b}}$ Derived from the ROC analysis.

${ }^{c}$ Youden index (sensitivity + specificity -1 ) is provided for an easier comparison of the combined value of the sensitivity and specificity, e.g., the diagnostic accuracy of CSF and PET measures as dichotomized variables. The value is based on the unbiased cutoffs.

Similar to the present study, they showed that $A \beta 42 /$ $t$-tau was superior to $A \beta 42$ and $A \beta 42 / A \beta 40$.

A possible advantage of $A \beta$ PET over CSF $A \beta 42$ as an early marker for amyloid pathology is that $A \beta$ PET may be able to identify early region-specific pathology. However, this was not supported by our study, since the global $A \beta$ uptake performed similar compared to the best regional SUVRs (tables 2 and 4). Only one previous study has examined this and found similar results. ${ }^{19}$ The similar AUCs for the best PET regions support the notion that the $A \beta$ deposition is uniformly distributed in the neocortical association areas already at the MCI stage of AD. ${ }^{35}$

We used classification cutoffs established with mixture modeling, which is a robust way of determining unbiased thresholds and used in several studies. ${ }^{20,36,37}$ Even so, the cutoffs (table 3) should not be considered generalizable, but study-specific for comparative purposes. However, figure 1 shows that although a cutoff is not optimized for the current population, it can still provide good diagnostic accuracy because of the broad range of high YI. The stability of cutoffs between populations is also supported by a previous cross-validation study on CSF A $\beta 42$ and amyloid PET cutoffs. ${ }^{20}$ However, even though the classification accuracy stays the same, a change in cutoff will of course result in a higher sensitivity/lower specificity or lower sensitivity/higher specificity, and this must be taken into consideration depending on the clinical aim of the examination.

The overall results were similar between the BioFINDER and ADNI cohorts. In ADNI, the same comparable results between regional and composite SUVRs, as well as equal diagnostic accuracies of CSF and PET measures, were seen (table 4). This similarity between studies is especially interesting considering the use of different PET tracers and different CSF assays. In both cohorts, numerically higher AUCs were seen for the CSF $A \beta 42 / t$-tau or $p$-tau ratios compared with just $A \beta 42$, but in $A D N I$ the increase was not significant (tables 2 and 4). This could be attributed to the poorer AUCs of t-tau and p-tau in ADNI (0.81 and 0.82; AlzBio3) compared with BioFINDER (0.88 and 0.87; EUROIMMUN and INNOTEST). A similar difference between INNOTEST and AlzBio3 regarding A $342 /$ tau ratios was also found in a previous study. ${ }^{38}$ It was notable that the AUCs of all brain regions were similar in ADNI (AUC range 0.01; table 4), in contrast to BioFINDER (AUC range 0.17; table 2). The reason for this could be that in ADNI the regions were coarser and not able to detect differences between, e.g., the medial and lateral temporal lobe.

The diagnostic accuracy of A $\beta$ PET and CSF biomarkers to detect incipient $\mathrm{AD}$ has only been compared head-to-head in one previous study, which partly used the same ADNI data used for replication in the present study. ${ }^{19}$ In that study, the accuracy between stable MCI (2- to 3-year follow-up without progression) and MCI$\mathrm{AD}$ was compared. In the present study, we instead compared healthy elderly and patients with MCI-AD, which resulted in higher AUCs (on average about 0.05 in the ADNI study; compare reference 19 and table 4). The rationale behind comparing controls and MCI-AD is that $>5-10$ years of follow-up is required before one can say that a patient with $\mathrm{MCI}$ is truly stable. ${ }^{36}$ Among patients with stable $\mathrm{MCI}$ with a short follow-up time, there are several cases with early-stage $\mathrm{AD}$. These patients with stable MCI will in most cases be correctly identified as MCI-AD by the biomarkers, but result in 
false low specificity (and a false low AUC) due to the incorrect clinical diagnosis/short follow-up. We therefore compared patients with MCI-AD and controls, given the relatively short follow-up data in the ADNI and BioFINDER populations, for a more robust comparison of $A \beta$ biomarkers.

The novelties of the present study compared with the previous study ${ }^{19}$ include a comparison between MCI-AD and controls, a more detailed analysis of regional $A \beta$ PET data, analyses of ratios of CSF $\mathrm{A} \beta 42 / \mathrm{A} \beta 40, \mathrm{~A} \beta 42 / \mathrm{t}$-tau, and $\mathrm{A} \beta 42 / \mathrm{p}$-tau, a comparison of 2 different ELISAs for CSF $A \beta 42$, and evaluation of the combination of PET and CSF biomarkers.

The similar results we found for CSF biomarkers and amyloid PET suggest that other factors than their diagnostic accuracy may be considered when deciding which biomarker to use. CSF analysis has the advantages that it may easily incorporate other biomarkers to improve the differential diagnosis (e.g., leukocytes, albumin ratio, neurofilament, $\alpha$-synuclein), requires less advanced instruments than PET, and is in some countries more available in clinical practice. Amyloid PET, on the other hand, is less invasive and has a higher reliability in longitudinal examinations and between centers. With appropriate standardized procedures, ${ }^{20,39,40} \mathrm{CSF}$ analysis and amyloid PET perform equally well and either method can be used in the clinical workup of $\mathrm{AD}$ for increased diagnostic accuracy.

\section{AUTHOR AFFILIATIONS}

From the Clinical Memory Research Unit, Department of Clinical Sciences (S.P., L.M., O.H.), and the Department of Computational Biology and Biological Physics (M.O.), Lund University; the Department of Neurology (S.P.) and the Memory Clinic (L.M., O.H.), Skåne University Hospital; the Clinical Neurochemistry Laboratory (H.Z., N.M., K.B.), Institute of Neuroscience and Physiology, the Sahlgrenska Academy at the University of Gothenburg, Mölndal, Sweden; the UCL Institute of Neurology (H.Z.), London, UK; the Department of Radiology and Biomedical Imaging (N.M.), University of California, San Francisco; the Department of Veterans Affairs Medical Center (N.M.), Center for Imaging of Neurodegenerative Diseases, San Francisco, CA; and the Department of Internal Medicine (P.J.), Sahlgrenska Academy, University of Gothenburg, Sweden.

\section{AUTHOR CONTRIBUTIONS}

Sebastian Palmqvist: drafting/revising the manuscript, study concept or design, analysis or interpretation of data, accepts responsibility for conduct of research and final approval, statistical analysis, study supervision. Henrik Zetterberg: drafting/revising the manuscript, analysis or interpretation of data, accepts responsibility for conduct of research and final approval, acquisition of data, obtaining funding. Niklas Mattsson: drafting/revising the manuscript, analysis or interpretation of data, accepts responsibility for conduct of research and final approval. Per Johansson: drafting/revising the manuscript, analysis or interpretation of data, accepts responsibility for conduct of research and final approval, acquisition of data, study supervision. Lennart Minthon: drafting/revising the manuscript, accepts responsibility for conduct of research and final approval, obtaining funding. Kaj Blennow: drafting/revising the manuscript, study concept or design, accepts responsibility for conduct of research and final approval, study supervision, obtaining funding. Mattias Ohlsson: drafting/revising the manuscript, analysis or interpretation of data, accepts responsibility for conduct of research and final approval, statistical analysis. Oskar Hansson: drafting/revising the manuscript, study concept or design, analysis or interpretation of data, accepts responsibility for conduct of research and final approval, contribution of vital reagents/ tools/patients, acquisition of data, study supervision, obtaining funding.

\section{ACKNOWLEDGMENT}

The authors thank the collaborators of this study and the entire BioFINDER study group (www.biofinder.se), including Ulf Andreasson, Susanna Vestberg for classifying the patients with MCI-AD into MCI subgroups, Erik Stomrud and Katarina Nägga for clinical evaluations of cognitively healthy individuals, Christer Nilsson for clinical evaluations of patients with mild cognitive symptoms, Per Wollmer and Douglas Hägerström for help with ${ }^{18} \mathrm{~F}$-flutemetamol PET imaging, Olof Lindberg for analyzing the hippocampal volumes, and Karin Nilsson, Rosita Nordkvist, Ida Friberg, Malin Otthén, and Johanna Fredlund for organizing inclusions and assessments.

\section{STUDY FUNDING}

Work in the authors' laboratory was supported by the European Research Council, the Swedish Research Council, the Strategic Research Area MultiPark (Multidisciplinary Research in Parkinson's disease) at Lund University, the Crafoord Foundation, the Swedish Brain Foundation, the Skåne University Hospital Foundation, the Swedish Alzheimer Association, Stiftelsen för Gamla Tjänarinnor, and the Swedish federal government under the ALF agreement. The Alzheimer's Disease Neuroimaging Initiative (ADNI) data collection and sharing for this project was funded by ADNI (NIH grant U01 AG024904) and DOD ADNI (Department of Defense award number W81XWH-12-2-0012). ADNI is funded by the National Institute on Aging, the National Institute of Biomedical Imaging and Bioengineering, and through contributions from the following: Alzheimer's Association; Alzheimer's Drug Discovery Foundation; Araclon Biotech; BioClinica, Inc.; Biogen Idec Inc.; Bristol-Myers Squibb Company; Eisai Inc.; Elan Pharmaceuticals, Inc.; Eli Lilly and Company; EuroImmun; F. HoffmannLa Roche Ltd and its affiliated company Genentech, Inc.; Fujirebio; GE Healthcare; IXICO Ltd.; Janssen Alzheimer Immunotherapy Research \& Development, LLC; Johnson \& Johnson Pharmaceutical Research \& Development LLC; Medpace, Inc.; Merck \& Co., Inc.; Meso Scale Diagnostics, LLC.; NeuroRx Research; Neurotrack Technologies; Novartis Pharmaceuticals Corporation; Pfizer Inc.; Piramal Imaging; Servier; Synarc Inc.; and Takeda Pharmaceutical Company. The Canadian Institutes of Health Research is providing funds to support ADNI clinical sites in Canada. Private sector contributions are facilitated by the Foundation for the NIH (www.fnih.org). The grantee organization is the Northern California Institute for Research and Education, and the study is coordinated by the Alzheimer's Disease Cooperative Study at the University of California, San Diego. ADNI data are disseminated by the Laboratory for NeuroImaging at the University of Southern California.

\section{DISCLOSURE}

S. Palmqvist, H. Zetterberg, N. Mattsson, P. Johansson, and L. Minthon report no disclosures relevant to the manuscript. K. Blennow has served at advisory boards for Koyowa Kirin Pharma, Eli Lilly, Pfizer, and Roche. Doses of ${ }^{18} \mathrm{~F}$-flutemetamol were sponsored by GE Healthcare. M. Ohlsson and O. Hansson report no disclosures relevant to the manuscript. Go to Neurology.org for full disclosures.

Received February 4, 2015. Accepted in final form June 3, 2015.

\section{REFERENCES}

1. Albert MS, DeKosky ST, Dickson D, et al. The diagnosis of mild cognitive impairment due to Alzheimer's disease: recommendations from the National Institute on Aging-Alzheimer's Association workgroups on diagnostic guidelines for Alzheimer's disease. Alzheimers Dement 2011;7:270-279.

2. Sperling RA, Aisen PS, Beckett LA, et al. Toward defining the preclinical stages of Alzheimer's disease: recommendations from the National Institute on Aging-Alzheimer's Association workgroups on diagnostic guidelines for Alzheimer's disease. Alzheimers Dement 2011;7:280-292. 
3. Blennow K, Zetterberg H, Haass C, Finucane T. Semagacestat's fall: where next for $\mathrm{AD}$ therapies? Nat Med 2013;19:1214-1215.

4. Eli Lilly and Company. Progress of Mild Alzheimer's Disease in Participants on Solanezumab Versus Placebo (EXPEDITION 3). In: ClinicalTrials.gov [Internet]. Bethesda, MD: National Library of Medicine. Cited January 20, 2015. Available at: https://clinicaltrials.gov/ct2/ show/NCT01900665. NLM Identifier: NCT01900665.

5. Karran E, Hardy J. Antiamyloid therapy for Alzheimer's disease: are we on the right road? N Engl J Med 2014;370: 377-378.

6. Seppala TT, Nerg O, Koivisto AM, et al. CSF biomarkers for Alzheimer disease correlate with cortical brain biopsy findings. Neurology 2012;78:1568-1575.

7. Wolk DA, Grachev ID, Buckley C, et al. Association between in vivo fluorine 18-labeled flutemetamol amyloid positron emission tomography imaging and in vivo cerebral cortical histopathology. Arch Neurol 2011;68:1398-1403.

8. Blennow K, Hampel H, Weiner M, Zetterberg H. Cerebrospinal fluid and plasma biomarkers in Alzheimer disease. Nat Rev Neurol 2010;6:131-144.

9. Buerger K, Ewers M, Pirttila T, et al. CSF phosphorylated tau protein correlates with neocortical neurofibrillary pathology in Alzheimer's disease. Brain 2006;129:3035-3041.

10. Degerman Gunnarsson M, Lindau M, Wall A, et al. Pittsburgh compound-B and Alzheimer's disease biomarkers in CSF, plasma and urine: an exploratory study. Dement Geriatr Cogn Disord 2010;29:204-212.

11. Fagan AM, Mintun MA, Mach RH, et al. Inverse relation between in vivo amyloid imaging load and cerebrospinal fluid Abeta42 in humans. Ann Neurol 2006;59:512-519.

12. Fagan AM, Mintun MA, Shah AR, et al. Cerebrospinal fluid tau and ptau(181) increase with cortical amyloid deposition in cognitively normal individuals: implications for future clinical trials of Alzheimer's disease. EMBO Mol Med 2009;1:371-380.

13. Forsberg A, Almkvist O, Engler H, Wall A, Langstrom B, Nordberg A. High PiB retention in Alzheimer's disease is an early event with complex relationship with CSF biomarkers and functional parameters. Curr Alzheimer Res 2010;7:56-66.

14. Grimmer T, Riemenschneider M, Forstl H, et al. Beta amyloid in Alzheimer's disease: increased deposition in brain is reflected in reduced concentration in cerebrospinal fluid. Biol Psychiatry 2009;65:927-934.

15. Jagust WJ, Landau SM, Shaw LM, et al. Relationships between biomarkers in aging and dementia. Neurology 2009;73:1193-1199.

16. Koivunen J, Pirttila T, Kemppainen N, et al. PET amyloid ligand [11C]PIB uptake and cerebrospinal fluid betaamyloid in mild cognitive impairment. Dement Geriatr Cogn Disord 2008;26:378-383.

17. Landau SM, Lu M, Joshi AD, et al. Comparing PET imaging and CSF measurements of $A \beta$. Ann Neurol 2013;74:826-836.

18. Mattsson N, Insel P, Donohue M, et al. Independent information from cerebrospinal fluid $\beta$-amyloid and florbetapir imaging in Alzheimer's disease. Brain 2015;138:772-783.

19. Mattsson N, Insel P, Landau S, et al. Diagnostic accuracy of CSF Ab42 and florbetapir PET for Alzheimer's disease. Ann Clin Transl Neurol 2014;1:534-543.

20. Palmqvist $S$, Zetterberg H, Blennow K, et al. Accuracy of brain amyloid detection in clinical practice using cerebrospinal fluid beta-amyloid 42: a cross-validation study against amyloid positron emission tomography. JAMA Neurol 2014;71:1282-1289.

21. Tolboom N, van der Flier WM, Yaqub M, et al. Relationship of cerebrospinal fluid markers to $11 \mathrm{C}$ $\mathrm{PiB}$ and 18F-FDDNP binding. J Nucl Med 2009;50: 1464-1470.

22. Weigand SD, Vemuri P, Wiste HJ, et al. Transforming cerebrospinal fluid Abeta42 measures into calculated Pittsburgh Compound B units of brain Abeta amyloid. Alzheimers Dement 2011;7:133-141.

23. McKhann GM, Knopman DS, Chertkow H, et al. The diagnosis of dementia due to Alzheimer's disease: recommendations from the National Institute on AgingAlzheimer's Association workgroups on diagnostic guidelines for Alzheimer's disease. Alzheimers Dement 2011;7:263-269.

24. Haftenberger M, Schuit AJ, Tormo MJ, et al. Physical activity of subjects aged 50-64 years involved in the European Prospective Investigation into Cancer and Nutrition (EPIC). Public Health Nutr 2002;5:1163-1176.

25. Nelissen N, Van Laere K, Thurfjell L, et al. Phase 1 study of the Pittsburgh compound B derivative 18F-flutemetamol in healthy volunteers and patients with probable Alzheimer disease. J Nucl Med 2009;50:1251-1259.

26. Lundqvist R, Lilja J, Thomas BA, et al. Implementation and validation of an adaptive template registration method for 18F-flutemetamol imaging data. J Nucl Med 2013;54: 1472-1478.

27. Rosen WG, Mohs RC, Davis KL. A new rating scale for Alzheimer's disease. Am J Psychiatry 1984;141:1356-1364.

28. Shaw LM, Vanderstichele H, Knapik-Czajka M, et al. Cerebrospinal fluid biomarker signature in Alzheimer's disease neuroimaging initiative subjects. Ann Neurol 2009;65:403-413.

29. Robin X, Turck N, Hainard A, et al. pROC: an opensource package for $\mathrm{R}$ and $\mathrm{S}+$ to analyze and compare ROC curves. BMC Bioinformatics 2011;12:77.

30. Benaglia T, Chauveau D, Hunter DR, Young D. Mixtools: an R package for analyzing finite mixture models. J Stat Softw 2009;32:1-29.

31. Fagan AM, Roe CM, Xiong C, Mintun MA, Morris JC, Holtzman DM. Cerebrospinal fluid tau/beta-amyloid(42) ratio as a prediction of cognitive decline in nondemented older adults. Arch Neurol 2007;64:343-349.

32. Hansson $\mathrm{O}$, Zetterberg $\mathrm{H}$, Buchhave $\mathrm{P}$, Londos $\mathrm{E}$, Blennow K, Minthon L. Association between CSF biomarkers and incipient Alzheimer's disease in patients with mild cognitive impairment: a follow-up study. Lancet Neurol 2006;5:228-234.

33. Hertze J, Minthon L, Zetterberg H, Vanmechelen E, Blennow K, Hansson O. Evaluation of CSF biomarkers as predictors of Alzheimer's disease: a clinical follow-up study of 4.7 years. J Alzheimers Dis 2010;21:1119-1128.

34. Tapiola T, Alafuzoff I, Herukka SK, et al. Cerebrospinal fluid \{beta\}-amyloid 42 and tau proteins as biomarkers of Alzheimer-type pathologic changes in the brain. Arch Neurol 2009;66:382-389.

35. Thal DR, Attems J, Ewers M. Spreading of amyloid, tau, and microvascular pathology in Alzheimer's disease: findings from neuropathological and neuroimaging studies. J Alzheimers Dis 2014;42:S421-S429.

36. Buchhave P, Minthon L, Zetterberg H, Wallin AK, Blennow K, Hansson O. Cerebrospinal fluid levels of 
beta-amyloid 1-42, but not of tau, are fully changed already 5 to 10 years before the onset of Alzheimer dementia. Arch Gen Psychiatry 2012;69:98-106.

37. De Meyer G, Shapiro F, Vanderstichele $H$, et al. Diagnosis-independent Alzheimer disease biomarker signature in cognitively normal elderly people. Arch Neurol 2010;67:949-956.

38. Le Bastard N, Coart E, Vanderstichele H, Vanmechelen E, Martin JJ, Engelborghs S. Comparison of two analytical platforms for the clinical qualification of Alzheimer's dis- ease biomarkers in pathologically-confirmed dementia. J Alzheimers Dis 2013;33:117-131.

39. Klunk WE, Koeppe RA, Price JC, et al. The Centiloid project: standardizing quantitative amyloid plaque estimation by PET. Alzheimers Dement 2015;11:1-15.e1-4.

40. Vanderstichele H, Bibl M, Engelborghs S, et al. Standardization of preanalytical aspects of cerebrospinal fluid biomarker testing for Alzheimer's disease diagnosis: a consensus paper from the Alzheimer's Biomarkers Standardization Initiative. Alzheimers Dement 2012;8:65-73.

\section{Enjoy Big Savings on NEW 2015 AAN Practice Management Webinar Subscriptions}

The American Academy of Neurology offers 14 cost-effective Practice Management Webinars for which you can attend live or listen to recordings posted online. AAN members can purchase one webinar for $\$ 149$ or subscribe to the entire series for only $\$ 199$ - a big savings from the 2015 nonmember price of $\$ 199$ per webinar or $\$ 649$ for the subscription. Register today for upcoming webinars, access recorded webinars, and see the rest of the 2015 schedule at AAN.com/view/pmw15:

- October 13: Fundamentals of E/M Coding

- November 10: Getting More Bang for Your Buck with Your EHR

\section{AAN Annual Meeting Upcoming Dates \& Deadlines}

Don't miss these important dates for the 2016 AAN Annual Meeting taking place April 15-21, 2016, in Vancouver, BC, Canada. Learn more at AAN.com/view/AM16.

- Abstract Submission Deadline: October 26, 2015

- Awards Application Deadline: October 28, 2015

- Registration Opens: November 2015 


\section{Neurology}

\section{Detailed comparison of amyloid PET and CSF biomarkers for identifying early Alzheimer disease}

Sebastian Palmqvist, Henrik Zetterberg, Niklas Mattsson, et al.

Neurology 2015;85;1240-1249 Published Online before print September 9, 2015

DOI 10.1212/WNL.0000000000001991

This information is current as of September 9, 2015

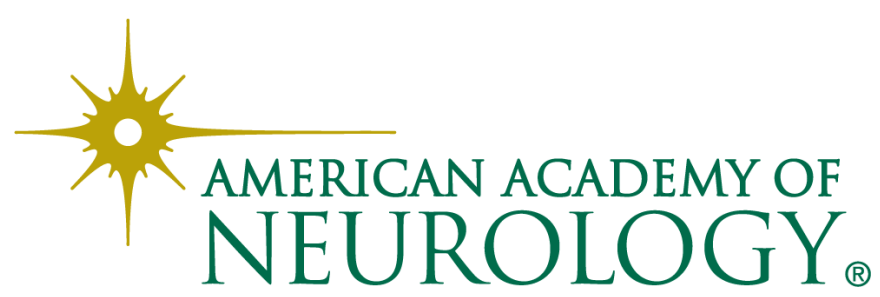




\section{Updated Information \& Services}

\section{Supplementary Material}

\section{References}

\section{Citations}

Subspecialty Collections

\section{Permissions \& Licensing}

\section{Reprints}

including high resolution figures, can be found at: http://n.neurology.org/content/85/14/1240.full

Supplementary material can be found at: http://n.neurology.org/content/suppl/2015/09/09/WNL.0000000000001 991.DC1

http://n.neurology.org/content/suppl/2015/09/30/WNL.0000000000001 991.DC2

http://n.neurology.org/content/suppl/2015/12/15/WNL.0000000000001 991.DC3

This article cites 39 articles, 6 of which you can access for free at: http://n.neurology.org/content/85/14/1240.full\#ref-list-1

This article has been cited by 13 HighWire-hosted articles: http://n.neurology.org/content/85/14/1240.full\#\#otherarticles

This article, along with others on similar topics, appears in the following collection(s):

\section{Alzheimer's disease}

http://n.neurology.org/cgi/collection/alzheimers_disease

Cerebrospinal Fluid

http://n.neurology.org/cgi/collection/cerebrospinal_fluid

Diagnostic test assessment

http://n.neurology.org/cgi/collection/diagnostic_test_assessment_

MCI (mild cognitive impairment)

http://n.neurology.org/cgi/collection/mci_mild_cognitive_impairment

\section{PET}

http://n.neurology.org/cgi/collection/pet

Information about reproducing this article in parts (figures,tables) or in its entirety can be found online at:

http://www.neurology.org/about/about_the_journal\#permissions

Information about ordering reprints can be found online:

http://n.neurology.org/subscribers/advertise

Neurology ${ }^{\circledR}$ is the official journal of the American Academy of Neurology. Published continuously since 1951, it is now a weekly with 48 issues per year. Copyright @ 2015 American Academy of Neurology. All rights reserved. Print ISSN: 0028-3878. Online ISSN: 1526-632X.

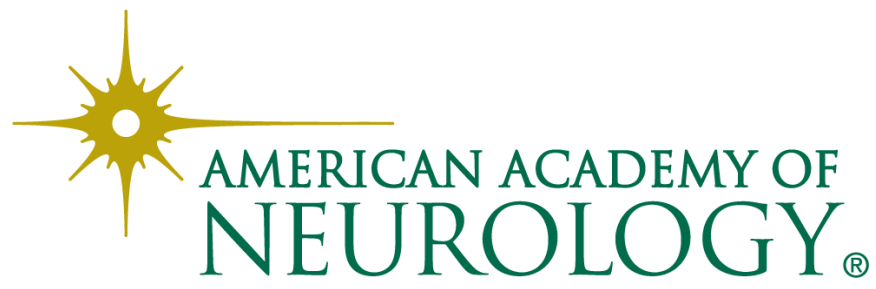

